$$
\bar{v}=\sigma^{2}\left[n-m+\frac{1}{\sigma^{2}}\left(\frac{u_{1}^{2}}{b_{11}}+\cdots+\frac{u_{m}^{2}}{b_{m m}}\right)\right] .
$$

Again we observe that the expected value of $v$ is $(n-m) \sigma^{2}$ when each of the $m$ linear forms is equated to zero. However, if $s$ of the $m$ linear forms are equated to their respective standard derivations while the remaining $m-s$ are equated to zero, then $\vec{v}=(n-m+s) \sigma^{2}$. Finally we see that the expected value of $v$, for a fixed set of $u$ 's, is not in general an integral multiple of $\sigma^{2}$.

The University of Iowa

\title{
ON THE PRESERVATION OF ANGLES AT A BOUNDARY POINT IN CONFORMAL MAPPING $\dagger$
}

\section{BY S. E. WARSCHAWSKI}

The object of this note is to prove the following theorem.

THEOREM. Let $R$ be a simply connected "schlicht" region in the $w$-plane whose boundary contains the point $w=0$. Let $w=0$ be "accessible" along the Jordan curve L. Suppose that there is a circle $|w|<\rho$ such that the part of the boundary of $R$ which is inside this circle lies within the angles

$$
\left|\arg w-h_{+}\right| \leqq k_{+}, \quad\left|\arg w-h_{-}\right| \leqq k_{-}, \quad\left(h_{-} \leqq h_{+}\right) .
$$

Suppose, furthermore, that $L$ connects $w=0$ with a boundary point outside $|w|=\rho$ such that $L$ divides $R$ into two sub-regions. Let all boundary points of one sub-region which are in $|w|<\rho$, and not on $L$, be in one of the angles (1), and those of the other sub-region which are in $|w|<\rho$, and not on $L$, be in the other.

Let $w=w(z)$ map $|z-1|<1$ conformally on $R$ in such a manner that its inverse function approaches 0 as $w \rightarrow 0$ along $L$. Let

$$
\begin{aligned}
& H(\alpha)=\frac{1}{\pi}\left[\left(\frac{\pi}{2}+\alpha\right) h_{+}+\left(\frac{\pi}{2}-\alpha\right) h_{-}\right], \\
& K(\alpha)=\frac{1}{\pi}\left[\left(\frac{\pi}{2}+\alpha\right) k_{+}+\left(\frac{\pi}{2}-\alpha\right) k_{-}\right] .
\end{aligned}
$$

$\dagger$ Presented to Society, October 26, 1935. 
Then

$$
-\epsilon-K(\arg z) \leqq \arg w(z)-H(\arg z) \leqq K(\arg z)+\epsilon,
$$

where $\epsilon \rightarrow 0$ as $z \rightarrow 0$ in any fixed angle $A_{\Theta}:|\arg z| \leqq \Theta<\pi / 2$.

Before proving this theorem we make the following remarks.

1. If the hypothesis of this theorem is satisfied for $k_{+}=k_{-}=k$, where $k$ is any arbitrary positive number, provided $\rho$ is small enough, then we say that the boundary of $R$ has a corner of measure $\beta=h_{+}-h_{-}$at $w=0$. In this case (3) means that

$$
\lim \arg \frac{w(z)}{z^{\beta / \pi}}
$$

exists as $z \rightarrow 0$ in any fixed angle $A_{\Theta}$.

If $\beta=\pi$, we shall say that the boundary of $R$ has a tangent at $w=0$.

2. Our theorem generalizes the well known result of $\mathrm{C}$. Carathéodory $\dagger$ and E. Lindelöf, $\ddagger$ namely, that the conformal representation of the interior of a Jordan curve on a circle is "quasiconform" at a boundary point with a corner of measure $\beta>0$, that is, the angles at this boundary point are transformed proportionally. This result has been extended recently by $\mathrm{J}$. Wolff $\S$ for $\beta=\pi$ for the case where the boundary is not necessarily a Jordan curve. In 1918 W. Gross $\|$ obtained a theorem which infers the result of our theorem from a less general hypothesis. I $A$ theorem analogous to ours for the case where the

$\dagger$ C. Carathéodory, Schwarz-Festschrift, pp. 40-41. See also Conformal Representation, Cambridge Tracts in Mathematics and Mathematical Physics, No. 28, pp. 91-93.

‡E. Lindelöf, Compte Rendu du quatrième congrès des mathématiciens Scandinaves, Stockholm, 1916, p. 87.

$\S$ J. Wolff, Comptes Rendus, vol. 200 (1935), pp. 42-43.

\| W. Gross, Mathematische Zeitschrift, vol. 2 (1918), p. 278.

If His hypothesis can be stated as follows (see loc. cit., pp. 276-277): Let $R$, the point $O(w=0)$ and $L$ have the same meaning as in our theorem. Draw a circle $c_{n}$ with center $O$ and radius $1 / n$, where $n \geqq n_{0}$ is so large that $c_{n}$ intersects the curve $L$. Follow $L$ from $O$ up to the first point of intersection $P_{n}$ with $c_{n}$. Let $\gamma_{n}$ denote the longest open arc of $c_{n}$ within $R$ containing $P_{n}$. Every "cut" $\gamma_{n},\left(n=n_{0}, n_{0}+1, \cdots\right)$, divides $R$ into two regions. Call $B_{n}$ that one which contains the part of $L$ from $O$ to $P_{n}$. Let $\Gamma_{n}$ be the part of the boundary of $B_{n}$ which $B_{n}$ has in common with $R$. According to Caratheodory's "Primenden" theory the accessible boundary points of $R$ can be arranged in a "cyclic order" 
boundary of $R$ in the neighborhood of $O$ is a Jordan curve has been given recently by A. Ostrowski. $\dagger$ All results mentioned above are contained as special cases in the theorem given in this note.

For the proof of our theorem we need the following lemma.

LEMma. Let $u(\zeta)$ be harmonic and bounded in the circle $|\zeta-1|<1$. Suppose that there exists an arc $\alpha_{+}$of $|\zeta-1|=1$, starting from $\zeta=0$, in the upper half-plane and an arc $\alpha_{-}$of $|\zeta-1|=1$, starting from $\zeta=0$, in the lower half-plane such that the radial boundary values $u^{*}(\zeta)$ of $u(\zeta)$ on $|\zeta-1|=1$, which exist for almost all $\zeta$ on $|\zeta-1|=1$, satisfy the conditions

$$
\left|u^{*}(\zeta)-h_{+}\right| \leqq k_{+} \text {on } \alpha_{+}, \quad\left|u^{*}(\zeta)-h_{-}\right| \leqq k_{-} \text {on } \alpha_{-},
$$

where $h_{+}, h_{-}, k_{+} \geqq 0$ and $k_{-} \geqq 0$ are given numbers. Then, if arg $\zeta$ is the branch which is 0 for $\zeta$ real and $>0$,

$$
-\epsilon-K(\arg \zeta) \leqq u(\zeta)-H(\arg \zeta) \leqq \epsilon+K(\arg \zeta)
$$

where $\epsilon \rightarrow 0$ as $\zeta \rightarrow 0$ in any way at all, and $K(\alpha)$ and $H(\alpha)$ are the functions given in (2).

Proof. The function

$$
P(\zeta) \equiv u(\zeta)-H(\arg \zeta)-K(\arg \zeta)
$$

is harmonic and bounded in $|\zeta-1|<1$. Because of (4) its boundary values $P^{*}(\zeta)$ on $|\zeta-1|=1$ which exist for almost all values of $\zeta$ on $|\zeta-1|=1$ satisfy the relation

$$
\varlimsup_{\zeta \rightarrow 0} P^{*}(\zeta) \leqq 0
$$

by means of the conformal representation of $R$. Gross requires now that all directions from $O$ to another accessible boundary point $P \neq O$ of $\Gamma_{n}$ for all $P$ "before" $O$ be within one of the angles (1) and for all $P$ "behind" $O$ be in the other, provided $n$ is sufficiently large. It is easily seen that, if this hypothesis is satisfied, then also that of our theorem holds. The converse is not necessarily true, as simple examples like the following show: $R$ is the circle $|w-1|<1$ without the following segments: (i) $\{\arg (w-1)=\pi / 2,3 / 4 \leqq|w-1| \leqq 1\}$ and (ii) $\left\{\pi-\pi /(2 n) \leqq \arg (w-1) \leqq \pi / 2,|w-1|=1-1 / n^{2}\right\},(n=2,3, \cdots)$; the boundary point in question is $w=0$ and $L$ is the radius at $w=0$. Our hypothesis is evidently satisfied with $h_{+}=-h_{-}=\pi / 2$ and any $k_{+}=k_{-}=k>0$, if $\rho$ is small enough. But the hypothesis of Gross is not fulfilled.

$\dagger$ Acta Mathematica, vol. 64 (1935), pp. 172-174. 
Furthermore, since $P(\zeta)$ is bounded, it can be represented by the Poisson integral with the boundary function $P^{*}(\zeta)$. Therefore, according to a theorem on Poisson's integral due to A. Ostrowski,

$$
\varlimsup_{\zeta \rightarrow 0} P(\zeta) \leqq 0
$$

as $\zeta \rightarrow 0$ in $|\zeta-1|<1$ in any way at all. $\dagger$

This proves the inequality on the right side of (5). That on the left is obtained by applying the same argument to $-[u(\zeta)-H(\arg \zeta)+K(\arg \zeta)]$.

Proof of the Theorem. 1. Let $S_{\rho}$ denote the largest connected subregion of $R$ and of $|w|<\rho$ containing the part of $L$ which is obtained when $L$ is described from $O(w=0)$ up to the first point of intersection $P$ with $|w|=\rho$. Call $a$ the longest arc of $|w|=\rho$ within $R$ containing $P ; a$ is part of the boundary of $S_{\rho}$. The region $S_{\rho}$ is simply connected. Therefore we can map the circle $|\zeta-1|<1$ on $S_{\rho}$ conformally by means of a function $w=\omega(\zeta)$ such that its inverse function approaches $O$ as $w \rightarrow 0$ along $L$. According to a well known theorem on correspondence at the boundary in conformal mapping, there is an arc $a^{\prime \prime}$ on $|\zeta-1|=1$ which contains $\zeta=0$ neither as an interior point nor as an end point, and which is mapped by $\omega(\zeta)$ on the arc $a$. The function $\omega(\zeta)$ thus has continuous boundary values on the open arc $a^{\prime \prime}$. Being bounded, $\omega(\zeta)$ assumes radial boundary values different from 0 at all other points of $|\zeta-1|=1$, except perhaps for a set of Lebesgue measure 0 . Since $\omega(\zeta)$ is univalent in $|\zeta-1|<1$, these boundary values are necessarily different from the interior points of $a$. Therefore they lie within the angles (1).

2 . Denote now by $\alpha_{+}$and $\alpha_{-}$the two arcs of $|\zeta-1|=1$, respectively, extending from $\zeta=0$ up to the end points of $a^{\prime \prime}$, the first starting in the upper, the second in the lower halfplane. Then we prove: For almost all points $\zeta=1+e^{i \theta}$ on $\alpha_{+}$, $\lim _{\rho \rightarrow 1} \omega\left(1+\rho e^{i \theta}\right)$ lies in the first of the angles (1), and for almost all $\zeta=1+e^{i \theta}$ on $\alpha_{-}$, this limit lies in the second of the angles (1). In order to establish this result, we need only show that if

† See A. Ostrowski, Jahresbericht der Deutschen Mathematiker-Vereinigung, vol. 36 (1927), p. 350. 
$\zeta^{\prime}$ and $\zeta^{\prime \prime}$ are two points on the same arc, say $\alpha_{+}$, at which the radial limits $w^{\prime}$ and $w^{\prime \prime}$ of $\omega(\zeta)$ exist and are different from 0 , then $w^{\prime}$ and $w^{\prime \prime}$ are in the same angle. Denote by $L^{\prime \prime}$ the image of the part $O P$ of $L$ within $S_{\rho}$ by means of the inverse function of $\omega(\zeta)$. The $\operatorname{arc} L^{\prime \prime}$ is a Jordan arc, ending at $\zeta=0$. Therefore we can find a point $\zeta_{1}^{\prime}$ on the radius at $\zeta^{\prime}$ and a point $\zeta_{1}^{\prime \prime}$ on the radius at $\zeta^{\prime \prime}$ and a Jordan arc $\gamma^{\prime}$ within $|\zeta-1|<1$ joining $\zeta_{1}^{\prime}$ and $\zeta_{1}^{\prime \prime}$, such that the curve $\gamma$, formed by $\gamma^{\prime}$ and the two straight segments $\zeta_{1}^{\prime} \zeta^{\prime}$ and $\zeta_{1}^{\prime \prime} \zeta^{\prime \prime}$, does not intersect $L^{\prime \prime}$. The image of $\gamma$ by means of $w=\omega(\zeta)$ is an arc within $S_{\rho}$ joining $w^{\prime}$ and $w^{\prime \prime}$, which does not intersect $L$. Therefore $w^{\prime}$ and $w^{\prime \prime}$ must be in the same angle.

3. Any branch of $\arg \omega(\zeta)$ is harmonic and bounded in $|\zeta-1|<1$. According to what we have said above, $\lim _{\rho \rightarrow 1}$ $\arg \omega\left(1+\rho e^{i \theta}\right)$ exists for almost all points on $|\zeta-1|=1$ and, if the branch is suitably chosen, we have

$$
\left|\lim _{\rho \rightarrow 1} \arg \omega\left(1+\rho e^{i \theta}\right)-h_{+}\right| \leqq k_{+} \text {on } \alpha_{+},
$$

and

$$
\left|\lim _{\rho \rightarrow 1} \arg \omega\left(1+\rho e^{i \theta}\right)-h_{-}\right| \leqq k_{-} \text {on } \alpha_{-} .
$$

Therefore we can infer from the preceding lemma:

(6) $-\eta-K(\arg \zeta) \leqq \arg \omega(\zeta)-H(\arg \zeta) \leqq K(\arg \zeta)+\eta$,

where $\eta \rightarrow 0$ as $\zeta \rightarrow 0$ in $|\zeta-1|<1$ in any way at all.

4. In order to obtain the connection between $\omega(\zeta)$ and $w(z)$ we first note that the inverse function of $w(z)$ maps $S_{\rho}$ on a subregion $S_{\rho}^{\prime}$ of the circle $|z-1|<1$ and the part $O P$ of $L$ on an arc $L^{\prime}$ ending at $z=0$. If $z(\zeta)$ is a suitable function which maps $|\zeta-1|<1$ on $S_{\rho}^{\prime}$ in such a manner that its inverse function approaches 0 as $z \rightarrow 0$ along $L$, then $\omega(\zeta)=w(z(\zeta))$. We shall establish a property of $z(\zeta)$ at $\zeta=0$.

The boundary of $S_{\rho}^{\prime}$ consists: (i) of a Jordan arc $a^{\prime}$ in $|z-1|<1$ which is the image of the arc $a$ of the boundary of $S_{\rho}$ and joins two points of $|z-1|=1$, different from each other and from $z=0$; (ii) of another point set $\Sigma_{\rho}^{\prime}$ in $|z-1| \leqq 1$, containing $z=0$. We prove that $S_{\rho}^{\prime}$ has a tangent at $z=0$ in the sense of the 
definition given in the first remark above. Let $\epsilon,(0<\epsilon<\pi / 2)$, be an arbitrarily given number. According to a well known theorem of Lindelöf, $*(z) \rightarrow 0$ uniformly in the angle $|\arg z| \leqq \pi / 2-\epsilon$. Therefore we can find a circle $C$ with center $z=0$ such that the image $\sigma$ of the sector $\sigma^{\prime}$, formed by this angle and an arc of $C$, by means of $w(z)$, lies inside $|w|<\rho$. Moreover, $\sigma$ is inside $S_{\rho}$, as can be seen in the following way. If at least one point of the arc $L^{\prime}$ is in $\sigma^{\prime}$, then $\sigma$ is evidently part of $S_{\rho}$. If, however, $\sigma^{\prime}$ does not contain any points of $L^{\prime}$, then we consider the closed Jordan curve, $\Gamma^{\prime}$, formed by a sub-arc $O Q$ of $L^{\prime}$, the radius $l$ at $z=0$, and an arc joining $Q$ and $z=1$ inside $|z-1|<1$. Since $\lim z_{j \rightarrow 0} w(z)=0$ on $L^{\prime}$ and on $l, w(z) \rightarrow 0$ uniformly as $z \rightarrow 0$ in the interior of $\Gamma^{\prime}$. $\dagger$ Hence, if we join a point $z_{1}$ of $L^{\prime}$ to a point $z_{2}$ of $l$ by an arc $\gamma^{\prime}$ within $\Gamma^{\prime}$, in a sufficiently small neighborhood of $z=0$, the image $\gamma$ will join $w\left(z_{1}\right)$ to $w\left(z_{2}\right)$ and will lie in $|w|<\rho$. Since $w\left(z_{2}\right)$ is a point in $\sigma$, this proves that $\sigma$ is in the same subregion of $R$ as the part $O P$ of $L$, that is, $\sigma$ is in $S_{\rho}$.

The fact that $\sigma$ is inside $S_{\rho}$ shows that all boundary points of $S_{\rho}^{\prime}$ are outside the sector $\sigma^{\prime}$. But since they certainly are within $|z-1| \leqq 1$, this proves the result about the tangent at $z=0$.

5. Now we observe that, if $\rho$ is replaced by a smaller number $r$, the region $S_{r}$ is a subregion of $S_{\rho}$ and therefore $S_{r}^{\prime}$ is a subregion of $S_{\rho}^{\prime}$. The boundary of $S_{r}^{\prime}$, as well as that of $S_{\rho}^{\prime}$, consists of a Jordan arc, joining two points of $|z-1|=1$, different from each other and from $z=0$, and of another point set $\Sigma_{r}^{\prime}$ containing $z=0$. The set $\Sigma_{r}^{\prime}$ is part of $\Sigma_{\rho}^{\prime}$. Furthermore, if $\rho \rightarrow 0$, $S_{\rho}^{\prime}$ is contracted to the point $z=0$. Therefore, given any $\epsilon$, $(0<\epsilon<\pi / 2)$, we can find $a \rho=\rho(\epsilon)$, such that for this $\rho$ the part $\Sigma_{\rho}^{\prime}$ of the boundary of $S_{\rho}^{\prime}$ lies inside the angles

$$
-\frac{\pi}{2} \leqq \arg z \leqq-\frac{\pi}{2}+\epsilon, \quad \text { and } \quad \frac{\pi}{2}-\epsilon \leqq \arg z \leqq \frac{\pi}{2} .
$$

We assume that a fixed number $\epsilon$ is given, and that $\rho$ and accordingly $S_{\rho}$ is chosen in such a manner that this condition is satisfied. Then $S_{\rho}^{\prime}$ evidently satisfies conditions at $z=0$ analo-

\footnotetext{
* See Acta Societatis Scientiarum Fennicae, vol. 46 (1915), no. 3, p. 10.

† See, for example, L. Bieberbach, Lehrbuch der Funktionentheorie, vol. 2,
} p. 21 . 
gous to those of $S_{\rho}$ at $w=0$ with $h_{+}=-h_{-}=\pi / 2, k_{+}=k_{-}=\epsilon$. Therefore, we can apply the relation (6) and obtain the result that, for a suitably chosen branch of $\arg z(\zeta)$ and $\arg \zeta$,

$$
\varlimsup_{\zeta \rightarrow 0}|\arg z(\zeta)-\arg \zeta| \leqq \epsilon
$$

as $\zeta \rightarrow 0$ in $|\zeta-1|<1$ in any way at all.

Let $\zeta=\zeta(z)$ denote the inverse function of $z(\zeta)$. Then $\zeta(z) \rightarrow 0$ as $z \rightarrow 0$ in any fixed angle $A_{\Theta}:|\arg z| \leqq \Theta<\pi / 2$ within $S_{\rho}^{\prime}$. From this and (7) it follows easily that also

$$
\varlimsup_{z \rightarrow 0}|\arg \zeta(z)-\arg z| \leqq \epsilon
$$

as $z \rightarrow 0$ in any fixed angle $A_{\Theta}$.

6. By observing that $w(z(\zeta))=\omega(\zeta)$ we obtain for $z$ in $A_{\Theta}$

$$
\begin{aligned}
& \varlimsup_{z \rightarrow 0}[\arg w(z)-H(\arg z)-K(\arg z)] \\
& \leqq \varlimsup_{\zeta \rightarrow 0}[\arg \omega(\zeta)-H(\arg \zeta)-K(\arg \zeta)] \\
&+\varlimsup_{z \rightarrow 0}|H(\arg (\zeta(z)))-H(\arg z)| \\
&+\varlimsup_{z \rightarrow 0}|K(\arg \zeta(z))-K(\arg z)| .
\end{aligned}
$$

From (6) and (8) it follows that

$$
\begin{gathered}
\varlimsup_{z \rightarrow 0}[\arg w(z)-H(\arg z)-K(\arg z)] \\
\leqq \frac{\epsilon}{\pi}\left[\left(h_{+}-h_{-}\right)+\left|k_{+}-k_{-}\right|\right] .
\end{gathered}
$$

In the same way we obtain

$$
\begin{aligned}
\varliminf_{z \rightarrow 0} & {[\arg w(z)-H(\arg z)+K(\arg z)] } \\
& \geqq-\frac{\epsilon}{\pi}\left[\left(h_{+}-h_{-}\right)+\left|k_{+}-k_{-}\right|\right] .
\end{aligned}
$$

Since this holds for any $\epsilon>0$ and the left side is independent of $\epsilon$, we can let $\epsilon$ tend to 0 and obtain the result (3).

School of Electrical EngineEring,

CORNELl University 